\title{
Influence of Lymphangio Vascular (V) and Perineural (N) Invasion on survival of patients with resected Esophageal Squamous Cell Carcinoma (ESCC): a single-center retrospective study
}

\author{
Chengke Xie ${ }^{\text {Equal first author, } 1}$, Zhiyao Chen ${ }^{\text {Equal first author, } 2}{ }^{2}$, Jie Xu ${ }^{2}$, Zhiyong Meng ${ }^{3}$, Zhijun Huang ${ }^{\text {Corresp., } 2}$, Jianqing Lin ${ }^{\text {Corresp. } 4}$ \\ ${ }^{1}$ Department of Gastrointestinal \& Esophageal Surgery, The Second Affiliated Hospital of Fujian Medical University, Quanzhou, China, 7 \\ 2 Department of Gastrointestinal \& Esophageal Surgery, The Second Affiliated Hospital of Fujian Medical University, Quanzhou, 34 Zhongshanbei Road, \\ Quanzhou 362000, Fujian, China., China \\ 3 Department of Ophthalmology, The Second Affiliated Hospital of Fujian Medical University, Quanzhou, 34 Zhongshanbei Road, Quanzhou 362000, Fujian, \\ China., China \\ 4 Department of Thyroid \& Breast Surgery, The Second Affiliated Hospital of Fujian Medical University, Quanzhou, 34 Zhongshanbei Road, Quanzhou \\ 362000, Fujian, China., China \\ Corresponding Authors: Zhijun Huang, Jianqing Lin \\ Email address: huangzj@fjmu.edu.cn, ljq13905977336@163.com
}

Abstract Background: Lymphangio vascular invasion (LVI) and perineural invasion (PNI) is associated with survival following resection for gastrointestinal cancer. But the relationship between LVI/PNI and survival of Esophageal Squamous Cell Carcinoma (ESCC) is still unclear. We aim to demonstrate the prognostic significance of LVI/PNI in ESCC.

Methods: A total of 195 ESCC patients underwent curative surgery from 2012 to 2018 was collected in the 2nd Affiliated Hospital of Fujian Medical University. All the patients were divided into four groups based on the status of the neurovascular invasion : (1) neither LVI nor PNI (VON0); (2) LVI alone (V1N0); (3) PNI alone (VON1); (4) combined LVI and PNI (V1N1) . First, the analysis included the Kaplan-Meier survival estimates with the Log rank test were performed to determine median OS in different groups divided according to the clinical factor, respectively. And the association between Overall Survival (OS) with multi clinical factors was examined using Cox regression analysis. Next, the risk factors for recurrence in patients with V1N1 were analyzed with univariate and multivariate analyses, respectively. Results: The cases in V0N0, V1N0, V0N1, and V1N1 groups were 91 (46.7\%), 62(31.8\%), 9 (4.6\%) and 33(16.9\%), respectively. The OS in the four groups was different $(P<0.001)$. The $1-, 3$ - and 5-year OS in V0N0 group was higher than that in V1N1 group, respectively (1-year OS: $93.4 \%$ vs $75.8 \%$, 3-year OS: $53.8 \%$ vs $24.2 \%, 5$-year OS: $48.1 \%$ vs $10.5 \%)$. The OS in stage I-II for patients with V1N1 was significantly lower than that in the other groups (V0N0, V1N0, V0N1) $(p<0.001)$. The postoperative adjuvant chemotherapy was a significant impact factor of OS for ESCC patients with V1N1 $(P=0.004)$. I vmphatic invasion and LVI were significantly prognosis Peer] reviewing PDF | (2021:08:64654:1:0:NEW 12'Jan 2022) 
factors associated $(\mathrm{P}=0.036, \mathrm{P}=0.030$, respectively). The ulcerative type is a risk factor for V1N1 occurance $(P=0.040)$. Conclusions: The $L V I$ and $P N I$ are important prognosis factors for ESCC patients. ESCC patients with simultaneous lymphangio vascular and perineural invasion (V1N1) showed worse OS than patients with either lymphangio vascular or perineural invasion alone (V1N0 or V0N1) or none (VON0). In addition, adjuvant chemotherapy may prolong the OS for ESCC patients with V1N1 . 
1 Influence of Lymphangio Vascular (V) and Perineural (N) Invasion on survival

2 of patients with resected Esophageal Squamous Cell Carcinoma (ESCC): a

3 single-center retrospective study

4 Chengke Xie*1, Zhiyao Chen*1, Jie Xu' ${ }^{1}$, Zhiyong Meng ${ }^{2}$, Zhijun Huang ${ }^{1}$, Jianqing Lin ${ }^{3}$

$6{ }^{1}$ Department of Gastrointestinal \& Esophageal Surgery, the 2nd Affiliated Hospital of Fujian

7 Medical University, Quanzhou, Fujian Province, 362000 China;

82 Department of Ophthalmology, the 2nd Affiliated Hospital of Fujian Medical University,

9 Quanzhou, Fujian Province, 362000 China;

$10{ }^{3}$ Department of Thyroid \& Breast Surgery, the 2nd Affiliated Hospital of Fujian Medical

11 University, Quanzhou, Fujian Province, 362000 China

$12 *$ : co-first authors

13 Corresponding authors:

14 Zhijun Huang: E-mail: huangzj@fjmu.edu.cn;

15 Jianqing Lin: E-mail: ljq13905977336@163.com

16 Contact information:

17 Chengke Xie E-mail: 18559305202@163.com

18 Zhiyao Chen E-mail: chenzyaoo@sina.com

19 Jie Xu E-mail: xj1310309221@126.com

20 Zhiyong Meng E-mail: biteoo@qq.com

Keywords: Esophageal squamous cell carcinoma, Lymphangio Vascular invasion, Perineural invasion $_{3}$, Overall Survival 4 , Prognosis $_{5}$

Abstract

Background: Lymphangio vascular invasion (LVI) and perineural invasion (PNI) is associated with survival following resection for gastrointestinal cancer. But the relationship between LVI/PNI 
and survival of Esophageal Squamous Cell Carcinoma (ESCC) is still unclear. We aim to demonstrate the prognostic significance of LVI/PNI in ESCC.

Methods: A total of 195 ESCC patients underwent curative surgery from 2012 to 2018 was collected in the 2nd Affiliated Hospital of Fujian Medical University. All the patients were divided into four groups based on the status of the neurovascular invasion: (1) neither LVI nor PNI (V0N0); (2) LVI alone (V1N0); (3) PNI alone (V0N1); (4) combined LVI and PNI (V1N1). First, the analysis included the Kaplan-Meier survival estimates with the Log rank test were performed to determine median Overall Survival (OS) in different groups divided according to the clinical factor, respectively. And the association between OS with multi clinical factors was examined using Cox regression analysis. Next, the risk factors for recurrence in patients with V1N1 were analyzed with univariate and multivariate logistic regression analyses, respectively.

Results: The cases in V0N0, V1N0, V0N1, and V1N1 groups were 91 (46.7\%), 62(31.8\%), 9 (4.6\%) and $33(16.9 \%)$, respectively. The OS in the four groups was different $(\mathrm{P}<0.001)$. The 1 , 3- and 5-year OS in V0N0 group was higher than that in V1N1 group, respectively (1-year OS: $93.4 \%$ vs $75.8 \%$, 3 -year OS: $53.8 \%$ vs $24.2 \%$, 5 -year OS: $48.1 \%$ vs $10.5 \%$ ). The OS in stage I-II for patients with V1N1 was significantly lower than that in the other groups (V0N0, V1N0, V0N1) $(\mathrm{p}<0.001)$. The postoperative adjuvant chemotherapy was a significant impact factor of OS for ESCC patients with V1N1 ( $\mathrm{P}=0.004)$. Lymphatic invasion and LVI were significantly prognosis factors associated $(\mathrm{P}=0.036, \mathrm{P}=0.030$, respectively). The ulcerative type is a risk factor for $\mathrm{V} 1 \mathrm{~N} 1$ occurance $(\mathrm{P}=0.040)$.

Conclusions: The LVI and PNI are important prognosis factors for ESCC patients. ESCC patients with simultaneous lymphangio vascular and perineural invasion (V1N1) showed worse OS than patients with either lymphangio vascular or perineural invasion alone (V1N0 or V0N1) or none (V0N0). In addition, adjuvant chemotherapy may prolong the OS for ESCC patients with V1N1.

INTRODUCTION

3 Esophageal cancer (EC), one of the most aggressive gastrointestinal malignancies, is the 4th most 
54

common cancer and the 4th leading cause of cancer-related mortality in China(Chen et al. 2016; Malhotra et al. 2017). And the main histologic type is squamous cell carcinoma in China, especially. Surgery has been the main curative treatment of resectable EC(Shah et al. 2020). However, the overall prognosis is still poor, particularly in ESCC: the five-year overall survival rate was only $15 \%$ to $25 \%$ (Pennathur et al. 2013). The node and metastasis (TNM) classification system were widely accepted to evaluate the prognosis of ESCC worldwide according to the American Joint Committee on Cancer (AJCC) guideline(Rice et al. 2017). The prediction of prognosis and survival rate of ESCC is based on the TNM system at present, but it is unable to evaluate accurately especially in the early stage(Cai \& Xin 2010; Rice et al. 2017). LVI and PNI could often be found in the postoperative pathological diagnosis of gastrointestinal tumors(Cao et al. 2020; Chen et al. 2020; Fang et al. ; Marx et al. 2020). Esophageal carcinoma cells directly infiltrate and spread through blood or lymphatic vessels and nerve invasion(Smyth et al. 2017). This pattern is the manifestation of invasive characteristics, which are often a precursor of tumor metastasis and disease progression(Smyth et al. 2017). Zhang et al had reported that microscopic lymphangio vascular or/and perineural invasion may be an important prognostic factor for ESCC(Zhang et al. 2018).

LVI which including micro-lymphatic vessel invasion (MLVI) and micro-blood vessel invasion (MBVI), is a risk factor of tumor metastasis and invasion(Gujam et al. 2014). Studies had found that the presence of LVI was an important factor for poor clinical prognosis patients with gastric cancer, colorectal cancer, ductal breast cancer, and non-small cell lung cancer(Fang et al. ; Gujam et al. 2014; Marx et al. 2020; Rurika et al. 2015). Similarly, LVI is also an independent risk factor of prognosis for patients with ESCC(Wang et al. 2016; Zhang et al. 2017).

PNI, which was known as perineural diffusion and neurotropism, is used to describe the process of cancer cells invading, surrounding, and passing through nerves(Batsakis 1985; Schmitd et al. 2018). PNI means that the changes of nerve cells and supporting cells in the background of cancer, including the changes and migration of the perineural matrix(Ekin et al. 2014). PNI could enhance the flexibility, mobility, and invasiveness of tumor cells, the injury, and regeneration of 
81

82

nerve cells \& the interaction, chemotaxis(Chen et al. 2019). PNI was not only the important pathway of tumor proliferation and metastasis, but also an independent risk factor affecting the of esophageal cancer prognosis(Kim et al. 2021; Zhang L 2021).

Compared to the traditional prognostic factors, the LVI and PNI may be the new risk factors for patients with ESCC, especially the lymph-node negative patients(Hsu et al. 2019; Kim et al. 2021). However, many studies only considered the influence of prognosis of LVI or PNI for ESCC patients, and ignored the relationship between the them, that may easily cause confusion and affect prognosis assessment. This study retrospectively analyzed the clinicopathological data of 195 ESCC patients with LVI or/and PNI, so as to provide a theoretical basis for clinicians to explore individualized treatment plans.

\section{MATERIALS AND METHODS}

\section{Patients and Characteristics}

A total of 195 ESCC patients who underwent radical esophagectomy with two- or three- field lymphadenectomy was collected in our center from Jan 2012 to Dec 2018. The inclusion criteria were as follows: (1) without neoadjuvant chemotherapy, radiotherapy and targeted therapy in preoperation; (2) one primary tumor only and no distant metastasis (M1) before surgery; (3) the histologic type was squamous cell carcinoma; (4) complete data including age, gender, TNM classification system (AJCC, 7th edition), pathologic information and follow up data. The study's retrospective protocol obtained the consent of patients by telephone and filled in informed consent and had been approved by the Medical ethics committee of the Second Affiliated Hospital of Fujian Medical University. (IRB number 124, 2021).

All the patients were divided into four groups according to the status of the neurovascular invasion: (1) neither LVI nor PNI (V0N0); (2) LVI alone (V1N0); (3) PNI alone (V0N1); (4) combined LVI and PNI (V1N1).

The evaluation indexes included age, gender, postoperative adjuvant chemotherapy, tumor location, tumor length, tumor gross specimen type, Stage, the depth of tumor invasion, lymphatic invasion, LVI, and PNI. 
109 Follow-Up

110 All the patients underwent CT-scan and hematological exam for personal interview every 3-6

111

112

113

114

115

116

117

118

119

120

121

122

123

124

125

126

127

128

129

130

131

132

133

134

months for post-operative Year 1-2, every 6-12 months for post-operative Year 3-5, and then every year beyond post-operative Year 5. The end-point event was patient death. The data on the patients without end-point events were censored until May 2021. The data for the patients who did not complete follow-up were censored at the last follow-up date.

\section{Pathological Examination}

All samples were reviewed by a team of experienced pathologists who know nothing about the patient's data for histological diagnosis and grade. The specimens were stained with hematoxylineosin (H \& E) and examined(Kim et al. 2021; Wang et al. 2016). LVI was detected as the presence of tumor cell clusters in vessels stained positive(Perry et al. 2015). And PNI was detected as the presence of tumor cells within any of the three layers of the nerve sheath, or near close to the nerve and involving $\geq 33 \%$ of the nerve circumference(Liebig et al. 2010). The microscopic sections of the four histological modes are shown in Figure 1.

\section{STATISTICAL ANALYSIS}

Statistical analyses were applied with SPSS software version 25.0 (IBM, SPSS Statistics, Chicago, IL, USA). The differences and cumulative hazard of OS were conducted using the Kaplan-Meier method and log-rank test. The relationships of OS with characteristics (sex, age, tumor site, tumor length, tumor gross specimen type, grade, lymphatic invasion, stage, depth of tumor invasion, LVI, PNI, and postoperative adjuvant chemotherapy) were determined using univariable and multivariable Cox proportional hazards model. The optimal cut points for variables identified as influencing V1N1 were confirmed using receiver operating characteristic (ROC) curve analysis. P $<0.05$ was considered significant.

\section{RESULTS}

\section{Patient Characteristics and Prevalence of LVI and PNI}

All patients included $140(71.8 \%)$ males and $55(28.2 \%)$ females. The median age was 60 years 
135

136

137

138

139

140

141

142

143

144

145

146

147

148

149

150

151

152

153

154

155

156

157

158

159

160

161

old. The overall prevalence of the LVI and PNI was $48.7 \%$ and $21.5 \%$, respectively. The patients with neither LVI nor PNI accounted for 46.7\%. Patients with combined LVI and PNI accounted for $16.9 \%$. Demographic information and the association of LVI and PNI with clinicopathological variables were showed in Table 1.

\section{Relationship of OS with LVI or/and PNI}

As showed in Figure 4 (F), the prognosis of patients with neither LVI nor PNI was superior to that with either LVI or PNI. $(\mathrm{P}<0.001)$ The average OS was $67 \pm 4.4$ months $(95 \% \mathrm{CI}: 58.2-75.8)$

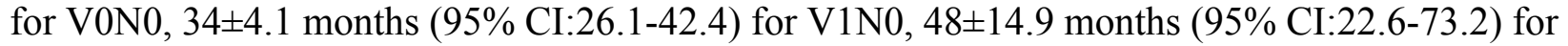
V0N1, and 32 \pm 5.1 months (95\% CI:22.3-42.5) for V1N1.

As showed in Figure 2, The OS in stage I-II for patients with V1N1 was significantly lower than that in the other stages (V0N0, V1N0, V0N1) $(\mathrm{p}<0.001)$. The OS was significantly difference in four groups for patients with negative lymphatic invasion $(p=0.005)$, but the OS was no significantly difference compared between patients with V1N0 and V0N1. And the OS in stage (T1-2) for patients with V1N0 and V1N1 was lower than that in other stages $(\mathrm{p}<0.001)$. Furthermore, the OS in stage(T3-4) for patients with V0N0 was higher than that with the other three groups (V1N0, V0N1, V1N1) $(\mathrm{p}=0.008)$. But the OS in stage (T3-4) was no significant difference among patients with V1N0, V1N1 and, V0N1, respectively.

The 1-, 3- and 5- year OS rates of the cohort subdivided by the status of neurovascular invasion were listed in Table 2. The 1-, 3- and 5- year OS for patients with V0N0 was significant higher than that with V1N1 (OS: $93.4 \%$ vs $75.8 \%, 53.8 \%$ vs $24.2 \%, 48.1 \%$ vs $10.5 \%$ ), respectively. (P $<0.001)$

\section{Postoperative Chemotherapy for Patients with LVI or/and PNI}

Of the 195 patients, $72(36.9 \%)$ patients including $23(11.8 \%)$ patients with V0N0, $25(12.8)$ with V1N0, 5 (2.6\%) with V0N1, and 19(9.7\%) with V1N1 underwent curative postoperative chemotherapy. The OS for patients who underwent curative postoperative chemotherapy with V1N1 was significantly lower than that with other three pathological groups (V1N0, V0N0, V0N1) $(p=0.004)$. But the OS was no significantly difference compared in subgroups for patients with 
162 V1N0, V0N0, and V0N1, respectively. (Figure 3).

163 Univariate and Multivariate Analysis of Survival

164 According to Cox regression univariate regression analysis, the results showed that the $\operatorname{sex}(\mathrm{p}=$ 165 0.036), stage category ( $p<0.001)$, lymphatic invasion $(\mathrm{p}<0.001)$, PNI $(\mathrm{p}=0.009)$, and LVI $(\mathrm{p}<$ 166 0.001) were closely associated with OS, as showed in Figure 4 (A-E). Multivariate analysis 167 showed that LVI $(\mathrm{P}=0.030)$ and lymphatic invasion $(\mathrm{P}=0.036)$ were independent risk factors 168 affecting the of ESCC prognosis, as showed in Table 3.

169 Risk Factors Related to Patients with V1N1

170 The gender, primary tumor length, gross specimen types, lymphatic invasion, and stage were the 171 predictive factors for patients with V1N1 using univariate logistic regression $(\mathrm{P}<0.05)$. 172 Multivariate logistic regression analysis showed that the Ulcerative type was only an independent 173 risk factor for ESCC patients with $\mathrm{V} 1 \mathrm{N1}(\mathrm{P}=0.04)$ (Table 4). And the AUC of the model was 0.821(CI: 0.752-0.890) using multivariate ROC analysis. Figure 4 (G).

175 176

\section{DISCUSSION}

Some studies had reported that the LVI is not only negative prognostic factor independent of tumor stage but also a high-risk factor of recurrence in ESCC patients(Hsu et al. 2019; Schoppmann et al. 2013; Semenkovich et al. 2020; Wang et al. 2016). The PNI was an adverse risk factor for survival in surgically treated patients with ESCC and reflected a more malignant behavior(Chen et al. 2014; Gao et al. 2016). And our results also showed that the LVI and PNI could be predicted factors for ESCC patients who underwent curative surgery. Our study had found that the presence of LVI and PNI were an important sign for poor clinical prognosis in lymph node negative patients. The results were the same as that in other studies(Chen et al. 2014; Gao et al. 2016; Huang et al. 2016; Ye et al. 2021). Especially, the OS in stage I-II for patients with V1N1 was significantly lower than those in the other groups (V1N0, V0N0, V0N1) $(\mathrm{p}<0.001)$.

Based on the 2019 edition of National Comprehensive Cancer Network (NCCN) guidelines, the LVI and/or PNI are only regarded as a risk of disease recurrence(Ajani et al. 2019). The postoperative adjuvant therapy was not recommended for the therapy for ESCC patients with LVI 
and/or PNI(Ajani et al. 2019). But the PNI is considered as an indicator of adjuvant radiotherapy for patients with head and neck tumors(Bakst et al. 2018). Some studies reported that PNI could be used as one of the reference indicators of adjuvant therapy for post-operative patients with esophageal cancer(C-Y et al. 2017; Gao et al. 2016). And some scholars also proposed that the LVI is an independently significant prognostic factor, and can be a potential determining factor for the use of adjuvant therapy for ESCC patients who underwent esophagogastrectomy (Schoppmann et al. 2013; Zafirellis et al. 2002). Interestingly, our results showed that postoperative adjuvant chemotherapy could prolong the overall survival of ESCC patients with simultaneous LVI and PNI (V1N1). Therefore, both LVI and PNI (V1N1) maybe an indicator for adjuvant therapy for postoperative ESCC patients. However, the prospective, multicenter, randomized controlled studies still need to be carried out to verify this conclusion.

The results showed that the sex, stage, lymphatic invasion, LVI and, PNI is the prognostic factor for ESCC patients, respectively $(\mathrm{P}<0.05)$. In addition, we found that the Lymphatic invasion and LVI were independent the prognostic factors for ESCC patients $(\mathrm{P}=0.036,0.030$, respectively). Therefore, we considered that the prognosis for postoperative ESCC patients could be more predicted accurately based on the TNM system and the status of the neurovascular invasion.

The logistic regression analysis results showed that the sex, depth of tumor invasion, primary tumor length, gross specimen type, lymphatic invasion, and stage were all risk factors $(\mathrm{P}<0.05)$ for ESCC patients with V1N1. But further analysis results showed that only the Ulcerative type is an independent risk factor $(\mathrm{P}=0.04)$. YJ Lee's study reported that ulcerative EGC exhibit more aggressive behaviors than non-ulcerative EGC concerning for to submucosal invasion, LNM, LVI, and PNI(Lee et al. 2016). A deep ulcer more than the thickness of the adjacent mucosal surface is an important factor for the invasion of submucosal carcinoma(Lee et al. 2012). Therefore, the ulcerative type may be a risk factor for the occurrence of both LVI and PNI.

Different for other studies, we have made a more detailed classification of the types of the neurovascular invasion, which is helpful to evaluate the prognosis more accurately. However, our 
216

217

218

219

220

221

222

223

224

225

226

227

228

229

230

231

232

233

234

235

236

237

238

239

240

241

242

243

244

research was a single-center retrospective study with the limited number of patients excluded that received neoadjuvant therapy. This is maybe a limitation in our research.

In conclusion, the OS and prognosis of ESCC patients with four pathological characteristics are different from each other, especially the patients in Stage I-II. The OS for patients with V1N1 is the worst compared with the other groups (V1N0, V0N1and V1N1). In addition, postoperative adjuvant chemotherapy could prolong the OS for ESCC patients with V1N1. Therefore, postoperative adjuvant chemotherapy could be recommended for the patients with V1N1. Besides, the ulcerative type may be a risk factor for the occurrence of both LVI and PNI. The LVI and PNI are important prognosis factors for ESCC patients.

\section{CONFLICTS OF INTEREST}

There is no conflict of interest.

\section{AUTHOR CONTRIBUTIONS}

Zhijun Huang and Jianqing Lin contributed to the conception of the study; Chengke Xie and Zhiyao Chen performed the data analyses and wrote the manuscript; Jie $\mathrm{Xu}$ and Zhiyong Meng helped perform the analysis with constructive discussions.

\section{FUNDING}

Natural Science Research Foundation of Fujian Province (No. 2019J01476), Medical Innovation Foundation of Fujian Province (No. 2019-CXB-19), High-level Talent Foundation of Quanzhou City (No. 2020C012R), and High-level Talent Innovation Foundation of Quanzhou City (No. 2017Z008).

\section{REFERENCES}

Ajani JA, D'Amico TA, Bentrem DJ, Chao J, Corvera C, Das P, Denlinger CS, Enzinger PC, Fanta P, Farjah F, Gerdes H, Gibson M, Glasgow RE, Hayman JA, Hochwald S, Hofstetter WL, Ilson DH, Jaroszewski D, Johung KL, Keswani RN, Kleinberg LR, Leong S, Ly QP, Matkowskyj KA, McNamara M, Mulcahy MF, Paluri RK, Park H, Perry KA, Pimiento J, Poultsides GA, Roses R, Strong VE, Wiesner G, Willett CG, Wright CD, McMillian NR, and Pluchino LA. 2019. Esophageal and Esophagogastric Junction Cancers, Version 2.2019, NCCN Clinical Practice Guidelines in Oncology. J Natl Compr Canc Netw DOI 17:855-883. 10.6004/jncen.2019.0033.

Bakst RL, Glastonbury CM, Parvathaneni U, Katabi N, and Yom SSJIJoRO. 2018. Perineural Invasion and Perineural 
Tumor Spread in Head and Neck Cancer: A Critical Review. Int J Radiat Oncol Biol Phys 103. DOI 10.1016/j.ijrobp.2018.12.009.

Batsakis JG. 1985. Nerves and neurotropic carcinomas. Ann Otol Rhinol Laryngol 94:426-427.

C-Y, Tsai, C-J, Yeh, Y-K, Chao, H-K, Chang, C-K, Oncology TJEJoSOtJotESoS, and Oncology tBAoS. 2017. Perineural invasion through the sheath in posttherapy esophagectomy specimens predicts poor survival in patients with esophageal squamous cell carcinoma. Eur $J$ Surg Oncol 43(10): 1-26 DOI 10.1016/j.ejso.2017.07.014.

Cai WJ, and Xin PL. 2010. Pattern of relapse in surgical treated patients with thoracic esophageal squamous cell carcinoma and its possible impact on target delineation for postoperative radiotherapy. Radiother Oncol 96:104-107. DOI 10.1016/j.radonc.2010.04.029.

Cao Y, Deng S, Yan L, Gu J, and Cai KJIJoCD. 2020. Perineural invasion is associated with poor prognosis of colorectal cancer: a retrospective cohort study. Int J Colorectal Dis 35(6) 1067-1075 DOI 10.1007/s00384020-03566-2.

Chen JW, Xie JD, Ling YH, Li P, Yan SM, Xi SY, Luo RZ, Yun JP, Xie D, and Cai MY. 2014. The prognostic effect of perineural invasion in esophageal squamous cell carcinoma. BMC Cancer 14:313. DOI 10.1186/14712407-14-313.

Chen L, Lin J, Chen LZ, Chen Y, Wang XJ, Guo ZQ, and Yu JM. 2020. Perineural Invasion and Postoperative Complications are Independent Predictors of Early Recurrence and Survival Following Curative Resection of Gastric Cancer. Cancer Manag Res 12:7601-7610. DOI 10.2147/CMAR.S264582.

Chen SH, Zhang BY, Zhou B, Zhu CZ, Sun LQ, and Feng YJJAJoCR. 2019. Perineural invasion of cancer: a complex crosstalk between cells and molecules in the perineural niche. Am J Cancer Res 9(1) 1-21.

Chen W, Zheng R, Baade PD, Zhang S, Zeng H, Bray F, Jemal A, Yu XQ, and He J. 2016. Cancer statistics in China, 2015. CA Cancer J Clin 66:115-132. DOI 10.3322/caac.21338

Ekin DI, Alexandra B, Pfitzinger PL, Steffen T, Eva B, Natascha K, Timo K, Matthias M, Marina L, and Melanie LJJotNCI. 2014. Investigation of Schwann Cells at Neoplastic Cell Sites Before the Onset of Cancer Invasion. J Natl Cancer Inst 106(8) 1-12 DOI 10.1093/jnci/dju184.

Fang LA, Zc B, Bt B, Yl A, Qz B, Lf B, Hd A, Ym A, Yong LBJP-R, and Practice. Influential factors and prognostic analysis of blood vessel invasion in advanced gastric cancer. Pathol Res Pract 216(3) 1-6 DOI 10.1016/j.prp.2019.152727.

Gao A, Wang L, Li J, Li H, Han Y, Ma X, and Sun Y. 2016. Prognostic Value of Perineural Invasion in Esophageal and Esophagogastric Junction Carcinoma: A Meta-Analysis. Dis Markers 2016:7340180. DOI $10.1155 / 2016 / 7340180$.

Gujam FJ, Going JJ, Mohammed ZM, Orange C, Edwards J, and McMillan DC. 2014. Immunohistochemical detection improves the prognostic value of lymphatic and blood vessel invasion in primary ductal breast cancer. $B M C$ Cancer 14:676. DOI 10.1186/1471-2407-14-676.

Hsu CP, Chuang CY, Hsu PK, Chien LI, and Wu YCJJoGS. 2019. Lymphovascular Invasion as the Major Prognostic Factor in Node-Negative Esophageal Cancer After Primary Esophagectomy. J Gastrointest Surg 24(7) 1-10 DOI 10.1007/s11605-019-04310-0.

Huang Q, Luo K, Chen C, Wang G, Jin J, Kong M, Li B, Liu Q, Li J, and Rong TJJoTO. 2016. Identification and Validation of Lymphovascular Invasion as a Prognostic and Staging Factor in Node-Negative Esophageal Squamous Cell Carcinoma. J Thorac Oncol 11:583-592. DOI 10.1016/j.jtho.2015.12.109. 
286

287

288

289

290

291

292

293

294

295

296

297

298

299

300

301

302

303

304

305

306

307

308

309

310

311

312

313

314

315

316

317

318

319

320

321

322

323

324

325

326

Kim HE, Park SY, Kim H, Kim DJ, and Kim SI. 2021. Prognostic effect of perineural invasion in surgically treated esophageal squamous cell carcinoma. Thorac Cancer 12:1605-1612. DOI 10.1111/1759-7714.13960.

Lee JI, Kim JH, Kim JH, Choi BJ, Song YJ, Choi SB, Bae YS, Lee SH, Jee SR, Kang MS, and Seol SY. 2012. Indication for endoscopic treatment of ulcerative early gastric cancer according to depth of ulcer and morphological change. J Gastroenterol Hepatol 27:1718-1725. DOI 10.1111/j.1440-1746.2012.07233.x

Lee YJ, Kim JH, Park JJ, Youn YH, Park H, Kim JW, Choi SH, Noh SH, and Green JJPO. 2016. The Implications of Endoscopic Ulcer in Early Gastric Cancer: Can We Predict Clinical Behaviors from Endoscopy? PLoS One 11(10) 1-12 DOI 10.1371/journal.pone.0164339.

Liebig C, Ayala G, Wilks JA, Berger DH, and Albo DJC. 2010. Perineural invasion in cancer: a review of the literature. Cancer 115:3379-3391. DOI 10.1002/cncr.24396.

Malhotra GK, Yanala U, Ravipati A, Follet M, Vijayakumar M, and Are C. 2017. Global trends in esophageal cancer. $J$ Surg Oncol 115:564-579. DOI 10.1002/jso.24592

Marx AH, Mickler C, Sauter G, Simon R, and Clauditz TSJIJoCD. 2020. High-grade intratumoral tumor budding is a predictor for lymphovascular invasion and adverse outcome in stage II colorectal cancer. Int J Colorectal Dis 35:1-10. DOI 10.1007/s00384-019-03478-w.

Pennathur A, Gibson MK, Jobe BA, and Luketich JD. 2013. Oesophageal carcinoma. Lancet 381:400-412. DOI 10.1016/S0140-6736(12)60643-6.

Perry C, Soomro I, Kaye P, Hardy E, Parsons SL, Ragunath K, Lobo DN, Martin SG, and Madhusudan S. 2015. Analysis of lymphatic and blood vessel invasion biomarkers in T1 esophagogastric adenocarcinomas for improved patient prognostication. Dis Esophagus 28:262-268. DOI 10.1111/dote.12190.

Rice TW, Gress DM, Patil DT, Hofstetter WL, Kelsen DP, and Blackstone EHJCACJfC. 2017. Cancer of the esophagus and esophagogastric junction-Major changes in the American Joint Committee on Cancer eighth edition cancer staging manual. CA Cancer J Clin 67 (4) 304-318 DOI 10.3322/caac.21399.

Rurika, Hamanaka, Tomoyuki, Yokose, Yuji, Sakuma, Masahiro, Tsuboi, Hiroyuki, and Pathology IJD. 2015. Prognostic impact of vascular invasion and standardization of its evaluation in stage I non-small cell lung cancer. Diagn Pathol 10 1-10 DOI 10.1186/s13000-015-0249-5.

Schmitd LB, Scanlon CS, and D'Silva NJ. 2018. Perineural Invasion in Head and Neck Cancer. J Dent Res 97:742750. DOI 10.1177/0022034518756297.

Schoppmann SF, Jesch B, Zacherl J, Riegler MF, Friedrich J, and Birner P. 2013. Lymphangiogenesis and lymphovascular invasion diminishes prognosis in esophageal cancer. Surgery DOI 153:526-534. DOI 10.1016/j.surg.2012.10.007.

Semenkovich TR, Yan Y, Subramanian M, Meyers BF, Kozower BD, Nava R, Patterson GA, Kreisel D, and Puri VJAoS. 2020. A Clinical Nomogram for Predicting Node-positive Disease in Esophageal Cancer. Ann Surg. 273(6) 1-8 DOI 10.1097/SLA.0000000000003450.

Shah MA, Kennedy EB, Catenacci DV, Deighton DC, Goodman KA, Malhotra NK, Willett C, Stiles B, Sharma P, Tang L, Wijnhoven BPL, and Hofstetter WL. 2020. Treatment of Locally Advanced Esophageal Carcinoma: ASCO Guideline. J Clin Oncol 38:2677-2694. DOI 10.1200/JCO.20.00866

Smyth EC, Lagergren J, Fitzgerald RC, Lordick F, Shah MA, Lagergren P, and Cunningham D. 2017. Oesophageal cancer. Nat Rev Dis Primers 3:17048. DOI 10.1038/nrdp.2017.48

Wang S, Chen X, Fan J, and Lu L. 2016. Prognostic Significance of Lymphovascular Invasion for Thoracic Esophageal Squamous Cell Carcinoma. Ann Surg Oncol 23:4101-4109. DOI 10.1245/s10434-016-5416-8.

Peer] reviewing PDF | (2021:08:64654:1:0:NEW 12 Jan 2022) 
327

328

329

330

331

332

333

334

335

336

337

338

339

340

341

342

343

344

345

346

347

348

349

350

351

352

353

354

355

356

357

358

Ye B, Zhang X, Su Y, Hao S, Teng H, Guo X, Yang Y, Sun Y, Mao T, and Li Z. 2021. The possibility of endoscopic treatment of cN0 submucosal esophageal cancer: results from a surgical cohort. Surg Endosc 35:593-601. DOI 10.1007/s00464-020-07420-y.

Zafirellis K, Dolan K, Fountoulakis A, Dexter SP, Martin IG, and Sue-Ling HM. 2002. Multivariate analysis of clinical, operative and pathologic features of esophageal cancer: who needs adjuvant therapy? Dis Esophagus 15:155-159. DOI 10.1046/j.1442-2050.2002.00230.x

Zhang H, Chen X, Wang S, Fan J, and Lu L. 2017. Poorer prognosis associated with simultaneous lymphatic and vascular invasion in patients with squamous carcinoma of the thoracic oesophagus. Eur J Cardiothorac Surg 52:378-384. DOI 10.1093/ejcts/ezx081.

Zhang L SJ, Liu Z, Pan J, Li B, Yang Y, He Y, Han Y, Li Z. 2021. ASO Visual Abstract:Occurrence and Prognostic Value of Perineural Invasion in Esophageal Squamous Cell Cancer: A Retrospective Study. Ann Surg Oncol. DOI 10.1245/s10434-021-10665-z.

Zhang WY, Chen XX, Chen WH, Zhang H, and Zou CL. 2018. Nomograms for predicting risk of locoregional recurrence and distant metastases for esophageal cancer patients after radical esophagectomy. BMC Cancer 18:879. DOI 10.1186/s12885-018-4796-5.

\section{Figure legend}

FIGURE 1. Pathological section of ESCC with four histological characteristics stained with hematoxylin and eosin: A: neither LVI nor PNI (V0N0); B: only LVI (V1N0); C: only PNI (V0N1); D: combined LVI and PNI (V1N1).

FIGURE 2. The prognosis of patients was determined in the four groups compared in subgroups (pathological stage, status of lymphatic invasion and pathological depth of tumor invasion). A: Stage I-II $p<0.001$; B: Stage III-IV p >0.05; C Ly (-) p =0.005; D Ly (+) p > 0.05; E: T1-2 p<0.001; F: T3-4 p=0.008.

FIGURE 3. Comparison of Kaplan-Meier curves stratified by whether postoperative adjuvant chemotherapy. A: Survival curve of Postoperative chemotherapy in V0N0 p=0.109; B: Survival curve of Postoperative chemotherapy in V1N0 p=0.933; C: Survival curve of Postoperative chemotherapy in V0N1 p=0.153; D: Survival curve of Postoperative chemotherapy in V1N1 $\mathrm{p}=0.004$

FIGURE 4. A-E: the prognostic impact of sex, stage, lymphatic invasion status and pattern of LVI and PNI on overall survival (OS). F: Mean survival analysis time in four pathological types, G: the value of statistically significant variables for ESCC patients with V1N1.

TABLE 1. Characteristics of Patients with ESCC ( $\mathrm{n}=195)$.

Peer] reviewing PDF | (2021:08:64654:1:0:NEW 12 Jan 2022) 
359 TABLE 2. Survival Rate in four pathological groups.

360 TABLE 3. Variables associate with overall survival in univariate and multivariate analysis for all patients.

361 TABLE 4. The Risk Factors associated with cancer specific survival using Chi square test, univariate and 362 multivariate logistic regression analysis. 
Table $\mathbf{1}$ (on next page)

Case baseline data of clinical study

Characteristics of Patients with ESCC ( $n=195)$ 


\begin{tabular}{|c|c|c|c|c|c|c|c|}
\hline \multirow[t]{2}{*}{ Clinical factors } & Median & \multirow[t]{2}{*}{ Variables } & Total & VONO & V1N0 & V0N1 & V1N1 \\
\hline & & & $\mathrm{n}=195$ & $\begin{array}{l}\mathrm{n}=91 \\
(46.7)\end{array}$ & $\begin{array}{l}\mathrm{n}=62 \\
(31.8)\end{array}$ & $\begin{array}{l}n=9 \\
(4.6)\end{array}$ & $\begin{array}{l}\mathrm{n}=33 \\
(16.9)\end{array}$ \\
\hline \multirow[t]{2}{*}{ Age year } & $60(41-78)$ & $<60(\%)$ & $92(47.2)$ & $41(21.0)$ & $27(13.8)$ & $6(3.1)$ & $18(9.2)$ \\
\hline & & $\geq 60(\%)$ & $103(52.8)$ & $50(25.6)$ & 35 (17.9) & $3(1.5)$ & $15(7.7)$ \\
\hline \multirow[t]{2}{*}{ Sex } & & Male (\%) & $140(71.8)$ & $58(29.7))$ & $46(23.6)$ & $7(3.6)$ & $29(14.9)$ \\
\hline & & Female (\%) & $55(28.2)$ & 33 (16.9) & $16(8.2)$ & $2(1.0)$ & $4(2.1)$ \\
\hline \multirow[t]{3}{*}{ Tumor location } & & Upper (\%) & $28(14.3)$ & $13(6.7)$ & $10(5.1)$ & $2(1.0)$ & $3(1.5)$ \\
\hline & & Middle (\%) & $97(46.7)$ & $56(28.7)$ & $28(14.3)$ & $2(1.0)$ & $11(5.6)$ \\
\hline & & Lower (\%) & $70(39.0)$ & $22(11.3)$ & $24(12.3)$ & $5(2.6)$ & $19(9.7)$ \\
\hline \multirow[t]{3}{*}{ Grade } & & Well (\%) & $36(18.5)$ & $16(8.2)$ & $9(4.6)$ & $3(1.5)$ & $8(4.1)$ \\
\hline & & Moderate (\%) & $121(62.0)$ & $61(31.3)$ & $40(20.5)$ & $5(2.6)$ & $15(7.7)$ \\
\hline & & Poor $(\%)$ & $38(19.5)$ & $14(7.2)$ & $13(6.7)$ & $1(0.5)$ & $10(5.1)$ \\
\hline \multirow[t]{4}{*}{ Depth of tumor invasion } & & T1 (\%) & $37(19.0)$ & $27(13.8)$ & $9(4.6)$ & 0 & $1(0.5)$ \\
\hline & & T2 (\%) & $36(18.5)$ & $24(12.3)$ & $10(5.1)$ & 0 & $2(1.0)$ \\
\hline & & T3 (\%) & $116(59.5)$ & $40(20.5)$ & $42(21.5)$ & $8(4.1)$ & $26(13.3)$ \\
\hline & & T4 (\%) & $6(3.0)$ & 0 & $1(0.5)$ & $1(0.5)$ & $4(2.1)$ \\
\hline \multirow[t]{2}{*}{ Primary tumor length $\mathrm{mm}$} & $36(8-100)$ & $<36(\%)$ & $104(53.3)$ & $61(31.3)$ & $34(17.4)$ & $3(1.5)$ & $23(11.8)$ \\
\hline & & $\geq 36(\%)$ & $91(46.7)$ & $30(15.4)$ & $28(14.4)$ & $6(3.1)$ & $10(5.1)$ \\
\hline \multirow[t]{3}{*}{ Gross specimen type } & & Other $(\mathbf{\%})$ & $60(30.8)$ & $42(21.5)$ & $12(6.2)$ & $2(1.0)$ & $2(1.0)$ \\
\hline & & $\begin{array}{c}\text { Ulcerative type } \\
\text { (\%) }\end{array}$ & $70(35.9)$ & $24(12.3)$ & $25(12.8)$ & $2(1.0)$ & $21(10.8)$ \\
\hline & & $\begin{array}{l}\text { Medullary type } \\
\text { (\%) }\end{array}$ & $65(33.3)$ & $25(12.8)$ & $25(12.8)$ & $5(2.6)$ & $10(5.1)$ \\
\hline \multirow[t]{4}{*}{ Lymphatic invasion } & & N0 (\%) & $91(46.7)$ & $70(35.9)$ & $9(4.6)$ & $7(3.6)$ & $5(2.6)$ \\
\hline & & N1 (\%) & $60(30.8)$ & $13(6.7)$ & $31(15.9)$ & 0 & $16(8.2)$ \\
\hline & & N2 (\%) & $33(16.9)$ & $7(3.6)$ & $16(8.2)$ & $1(0.5)$ & $9(4.6)$ \\
\hline & & N3 (\%) & $11(5.6)$ & $1(0.5)$ & $6(3.1)$ & $1(0.5)$ & $3(1.5)$ \\
\hline \multirow[t]{4}{*}{ Stage } & & I stage $(\mathbf{( \% )}$ & $28(14.3)$ & 24 (12.) & $3(1.5)$ & 0 & $1(0.5)$ \\
\hline & & II stage (\%) & $69(35.4)$ & $47(24.1)$ & $10(5.1)$ & 7 (3.6) & $5(2.6)$ \\
\hline & & III stage (\%) & $83(42.6)$ & $19(9.7)$ & $42(21.5)$ & 0 & $22(11.3)$ \\
\hline & & IV stage (\%) & $15(7.7)$ & $1(0.5)$ & $7(3.6)$ & $2(1.0)$ & $5(2.6)$ \\
\hline $\begin{array}{l}\text { Postoperative } \\
\text { chemotherapy }\end{array}$ & & Yes (\%) & 72 (36.9) & $23(11.8)$ & $25(12.8)$ & $5(2.6)$ & $19(9.7)$ \\
\hline
\end{tabular}


Table 2 (on next page)

Survival Rate in four pathological types

Survival Rate in four pathological types 


\begin{tabular}{llllll}
\hline Time & V0N0 & V1N0 & V0N1 & V1N1 & P-value \\
\hline 1-Year Survival Rate & $85 / 91=93.4$ & $46 / 69=66.7$ & $8 / 9=88.9$ & $25 / 33=75.8$ & 0.006 \\
3-Year Survival Rate & $49 / 91=53.8$ & $13 / 69=18.8$ & $2 / 9=22.2$ & $8 / 33=24.2$ & $<0.001$ \\
5-Year Survival Rate & $26 / 54=48.1$ & $5 / 32=15.6$ & $1 / 4=25$ & $2 / 19=10.5$ & 0.002 \\
\hline
\end{tabular}

1 


\section{Table 3(on next page)}

Comparison of survival for post-chemotherapy

Compared of OS for ESCC patients based on post-operative adjuvant chemotherapy in four pathological types. 
Table 3 Variables associate with overall survival in univariate and multivariate analysis for all patients

\begin{tabular}{|c|c|c|c|c|c|}
\hline \multirow{2}{*}{ Variable } & \multirow{2}{*}{ Ref } & \multicolumn{2}{|l|}{ univariate analysis } & \multicolumn{2}{|l|}{ multivariate analysis } \\
\hline & & $\mathrm{HR}(95 \% \mathrm{CI})$ & $\mathrm{p}$ value & $\mathrm{HR}(95 \% \mathrm{CI})$ & $\mathrm{p}$ value \\
\hline Age & $\geq 60$ & $1.164(0.799-1.697)$ & 0.428 & & \\
\hline Sex & male & $0.616(0.392-0.970)$ & 0.036 & $0.698(0.437-1.114)$ & 0.132 \\
\hline Tumor location & upper & & & & \\
\hline Middle & & $0.854(0.494-1.476)$ & 0.571 & & \\
\hline Lower & & $1.079(0.610-1.909)$ & 0.794 & & \\
\hline Grade & poor & & & & \\
\hline well & & $1.068(0.595-1.916)$ & 0.826 & & \\
\hline moderate & & $0.863(0.532-1.400)$ & 0.551 & & \\
\hline $\begin{array}{l}\text { Pathological depth of } \\
\text { tumor invasion }\end{array}$ & $\mathrm{T} 1-2$ & & & & \\
\hline $\mathrm{T} 3-4$ & & $1.469(0.983-2.197)$ & 0.061 & & \\
\hline Stage & stage I-II & & & & \\
\hline stage III-IV & & $2.169(1.470-3.201)$ & 0.000 & $0.658(0.301-1.437)$ & 0.293 \\
\hline Tumor length (mm) & $\geq 36$ & $1.287(0.885-1.871)$ & 0.186 & & \\
\hline Gross specimen type & Other type & & & & \\
\hline Ulcerative type & & $1.513(0.953-2.403)$ & 0.079 & & \\
\hline Medullary type & & $1.101(0.676-1.794)$ & 0.698 & & \\
\hline Lymphatic invasion & positive & $2.551(1.706-3.813)$ & 0.000 & $2.461(1.063-5.701)$ & 0.036 \\
\hline PNI & positive & $1.768(1.153-2.711)$ & 0.009 & $1.159(0.730-1.838)$ & 0.532 \\
\hline LVI & positive & $2.696(1.819-3.996)$ & 0.000 & $1.796(1.057-3.053)$ & 0.030 \\
\hline $\begin{array}{l}\text { Postoperative } \\
\text { chemotherapy }\end{array}$ & No & $0.924(0.626-1.364)$ & 0.692 & & \\
\hline
\end{tabular}




\section{Table 4 (on next page)}

The Risk Factors associated with V1N1

The Risk Factors associated with cancer specific survival using Chi square test, univariate and multivariate logistic regression analysis. 


\begin{tabular}{|c|c|c|c|c|c|c|c|c|c|c|c|}
\hline \multirow[t]{2}{*}{ Clinical factors } & \multirow[t]{2}{*}{ Variables } & \multirow[t]{2}{*}{ V1N1 } & \multirow{2}{*}{$\begin{array}{c}\text { No } \\
\text { V1N1 }\end{array}$} & \multirow[b]{2}{*}{$\mathrm{P}$} & \multirow[b]{2}{*}{$\chi^{2}$} & \multicolumn{3}{|c|}{ univariate analysis } & \multicolumn{3}{|c|}{ multivariate analysis } \\
\hline & & & & & & $\mathrm{P}$ & OR & $95 \% \mathrm{CI}$ & $\mathrm{P}$ & OR & $95 \% \mathrm{CI}$ \\
\hline \multirow[t]{2}{*}{ Age (years) } & $<60$ & 18 & 74 & 0.000 & 22.202 & & & & & & \\
\hline & $\geq 60$ & 15 & 88 & & & 0.354 & 0.701 & $\begin{array}{l}0.330- \\
1.486\end{array}$ & & & \\
\hline \multirow[t]{2}{*}{ Sex } & Male & 29 & 111 & 0.024 & 5.075 & & & & & & \\
\hline & Female & 4 & 51 & & & 0.032 & 0.300 & $\begin{array}{l}0.100- \\
0.899\end{array}$ & 0.078 & 0.346 & $\begin{array}{l}0.106- \\
1.125\end{array}$ \\
\hline \multirow[t]{3}{*}{ Tumor location } & Upper & 3 & 25 & 0.017 & 5.807 & & & & & & \\
\hline & Middle & 11 & 86 & & & 0.926 & 1.066 & $\begin{array}{l}0.276- \\
4.120\end{array}$ & & & \\
\hline & Lower & 19 & 51 & & & 0.090 & 3.105 & $\begin{array}{l}0.839- \\
11.487\end{array}$ & & & \\
\hline \multirow[t]{3}{*}{ Grade } & Well & 8 & 28 & 0.088 & 4.867 & & & & & & \\
\hline & Moderate & 15 & 106 & & & & & & & & \\
\hline & Poor & 10 & 28 & & & & & & & & \\
\hline \multirow{2}{*}{$\begin{array}{l}\text { Depth of tumor } \\
\text { invasion }\end{array}$} & $\mathrm{T} 1-2$ & 3 & 70 & 0.000 & 13.626 & & & & & & \\
\hline & T3-4 & 30 & 92 & & & 0.001 & 7.609 & $\begin{array}{l}2.231- \\
25.949\end{array}$ & 0.058 & 4.565 & $\begin{array}{l}0.950- \\
21.930\end{array}$ \\
\hline \multirow{2}{*}{$\begin{array}{l}\text { Primary tumor } \\
\text { length ( } \mathrm{mm} \text { ) }\end{array}$} & $<36$ & 10 & 94 & 0.004 & 8.465 & & & & & & \\
\hline & $\geq 36$ & 23 & 68 & & & 0.005 & 3.179 & $\begin{array}{l}1.421- \\
7.114\end{array}$ & 0.133 & 1.968 & $\begin{array}{l}0.814- \\
4.762\end{array}$ \\
\hline \multirow{3}{*}{$\begin{array}{l}\text { Histologic } \\
\text { types }\end{array}$} & Other & 3 & 57 & 0.000 & 29.995 & & & & & & \\
\hline & $\begin{array}{l}\text { Ulcerative } \\
\text { type }\end{array}$ & 20 & 50 & & & 0.002 & 7.600 & $\begin{array}{l}2.131- \\
27.104\end{array}$ & 0.040 & 4.302 & $\begin{array}{l}1.068- \\
17.332\end{array}$ \\
\hline & $\begin{array}{l}\text { Medullary } \\
\text { type }\end{array}$ & 10 & 55 & & & 0.070 & 3.455 & $\begin{array}{l}0.902- \\
13.224\end{array}$ & 0.301 & 2.172 & $\begin{array}{l}0.499- \\
9.462\end{array}$ \\
\hline \multirow{2}{*}{$\begin{array}{l}\text { Lymphatic } \\
\text { invasion }\end{array}$} & Negative & 5 & 86 & 0.000 & 15.851 & & & & & & \\
\hline & Positive & 28 & 76 & & & 0.000 & 6.337 & $\begin{array}{l}2.330- \\
17.231\end{array}$ & 0.052 & 10.960 & $\begin{array}{l}0.977- \\
122.917\end{array}$ \\
\hline \multirow[t]{2}{*}{ Stage } & I-II stage & 6 & 91 & 0.000 & 15.828 & & & & & & \\
\hline & III-IV stage & 27 & 71 & & & 0.000 & 5.768 & $\begin{array}{l}2.259- \\
14.728\end{array}$ & 0.399 & 0.357 & $\begin{array}{l}0.033- \\
3.914\end{array}$ \\
\hline
\end{tabular}


Figure 1

Microscopic features of four pathological patterns

Pathological section of ESCC with four histological characteristics stained with hematoxylin and eosin: A: neither LVI nor PNI (VON0); B: only LVI (V1N0); C: only PNI (VON1); D: combined LVI and PNI (V1N1)
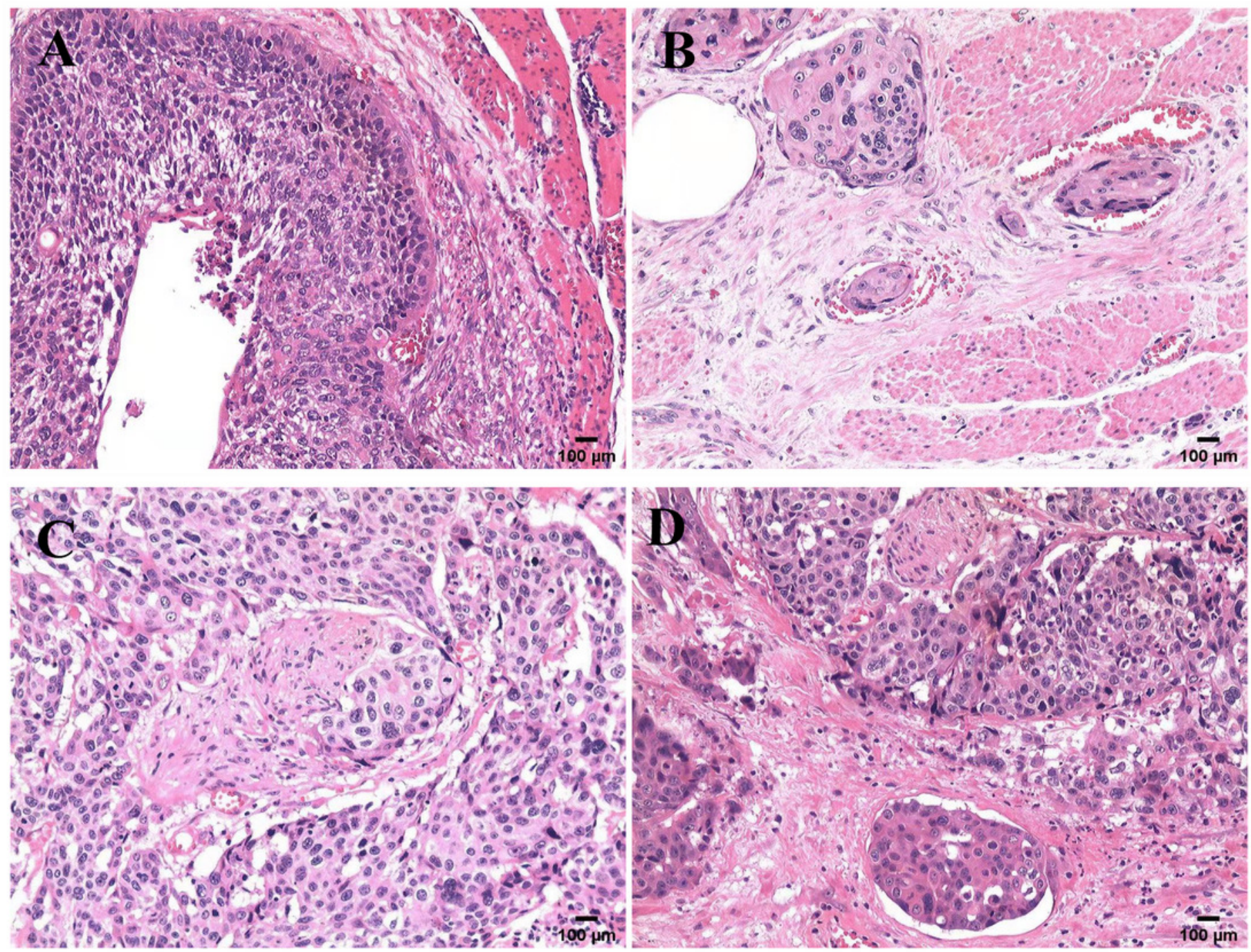
Figure 2

The prognosis of patients was determined in specific situation compared in subgroups

The prognosis of patients was determined in the four groups compared in subgroups (pathological stage, status of lymphatic invasion and pathological depth of tumor invasion). A: Stage I-II $p<0.001 ; B$ : Stage III-IV p>0.05; C Ly (-) $p=0.005$; D Ly (+) $p>0.05$; E: T1-2 $p<0.001 ; F:$ T3-4 $p=0.008$. 

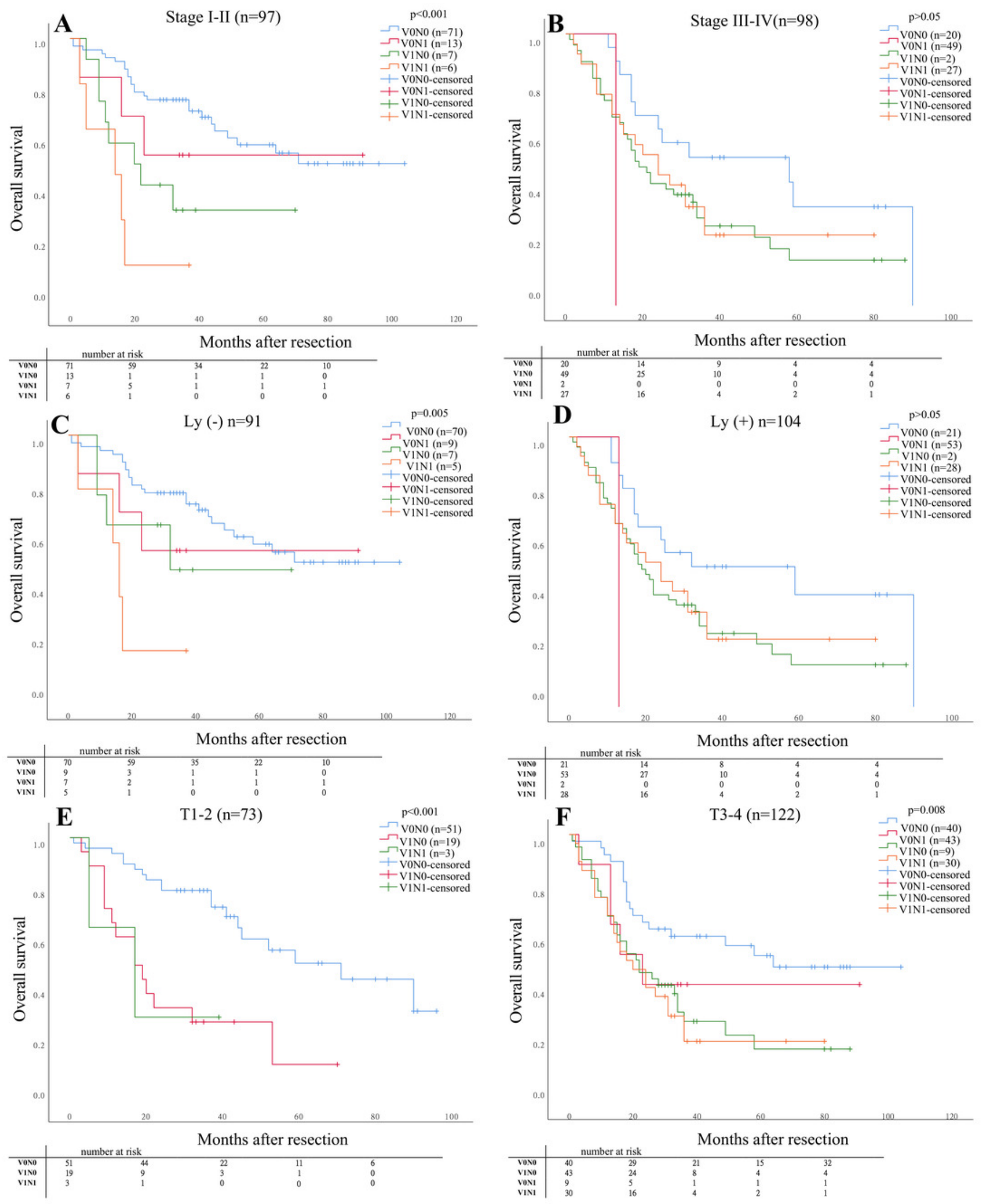
Figure 3

Comparison of Kaplan-Meier curves stratified by whether postoperative adjuvant chemotherapy

Comparison of Kaplan-Meier curves stratified by whether postoperative adjuvant chemotherapy. A: V0N0 $p=0.109 ; B$ : V1N0 $p=0.933$; V0N1 $p=0.153 ;$ V1N1 $p=0.004$
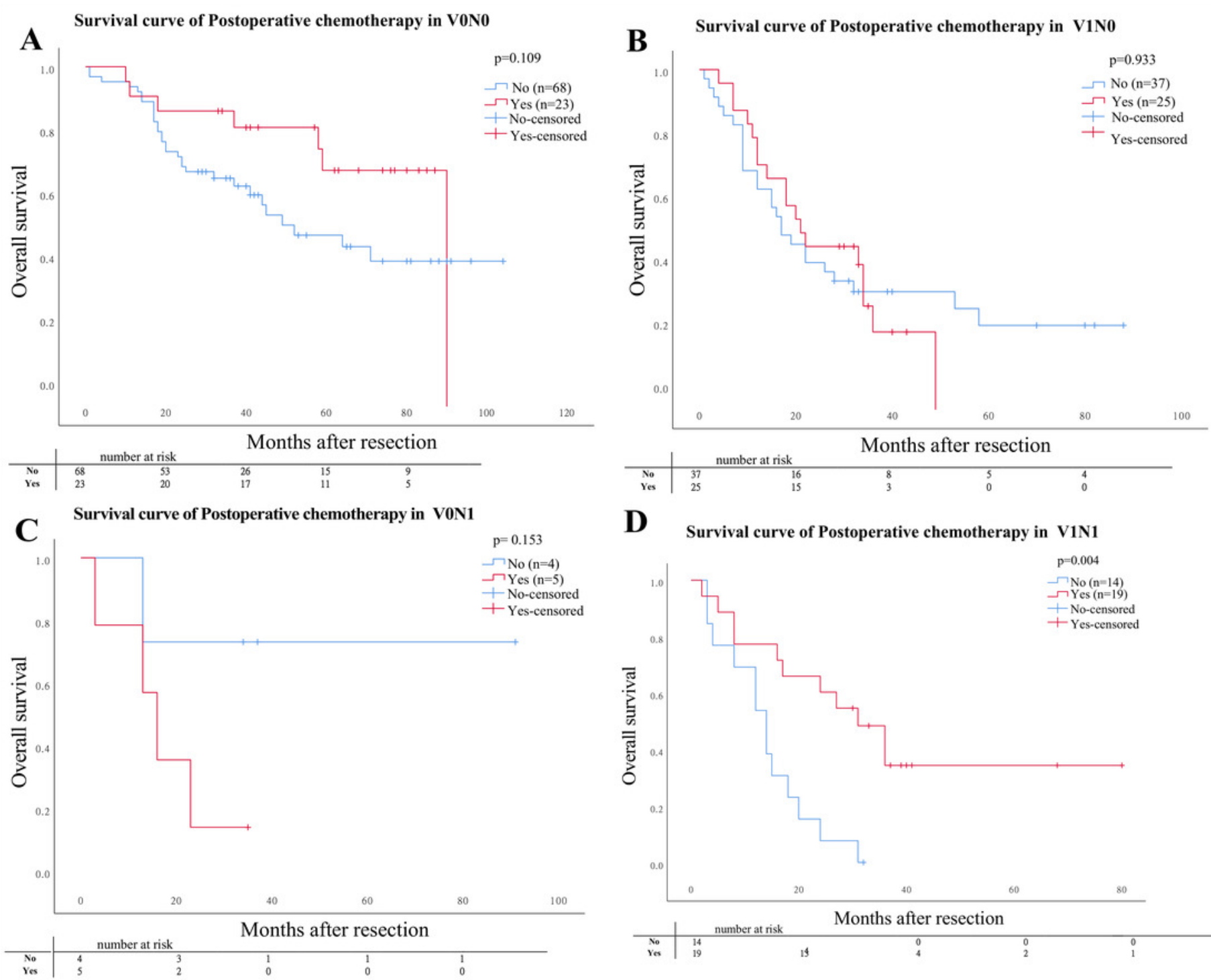

D Survival curve of Postoperative chemotherapy in V1N1
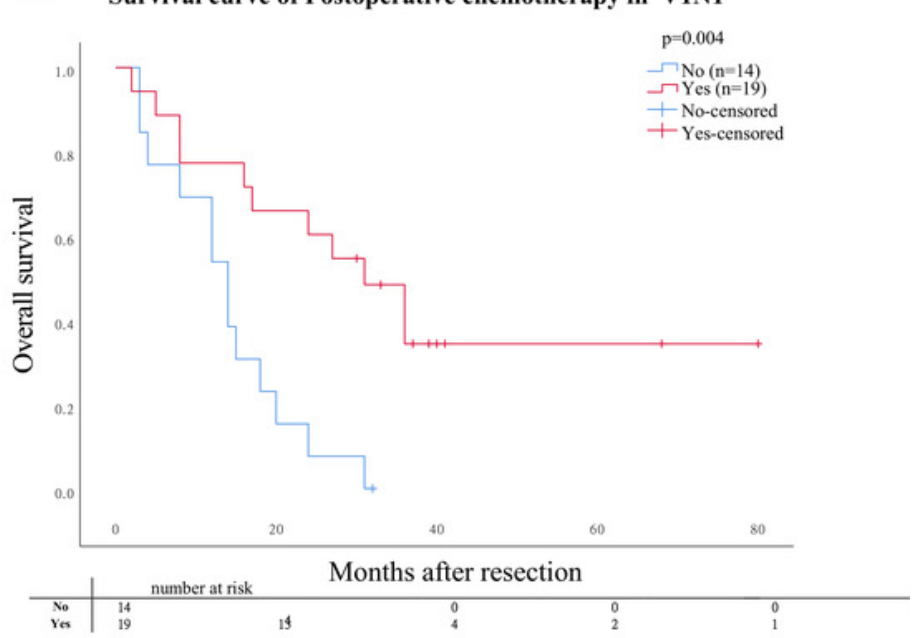


\section{Figure 4}

\section{Prognostic factors}

A-E: the prognostic impact of sex, stage, lymphatic invasion status and pattern of LVI and PNI on overall survival (OS). F: Mean survival analysis time in four pathological types, G: the value of statistically significant variables for ESCC patients with V1N1. 

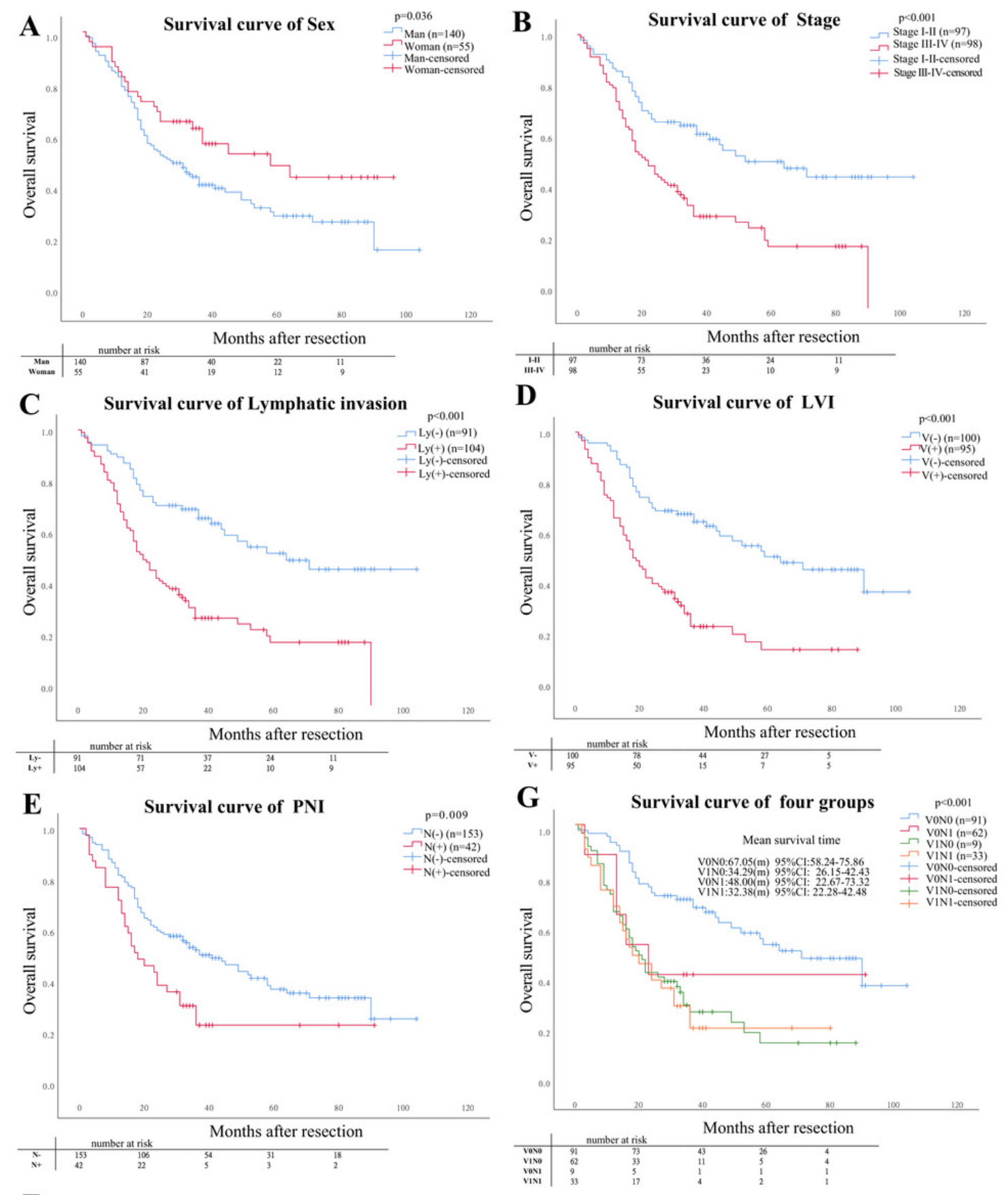

F

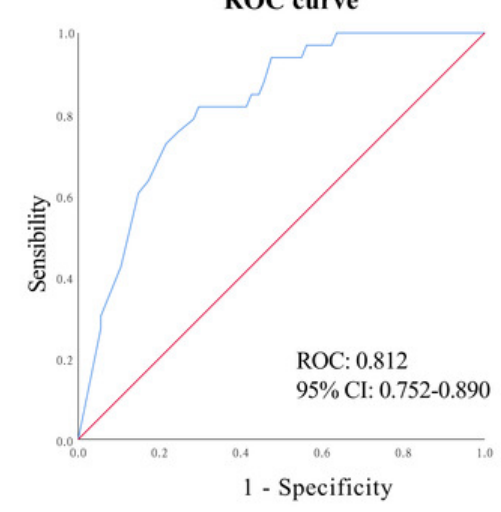

